\title{
RECUPERACIÓN Y TRATAMIENTO DE OPINIÓN MOVILIZADA PARA EL ANÁLISIS DEL CLIMA SOCIAL
}

\author{
Naiara Sagarminaga-Gutiérrez y Agustí Canals-Parera
}
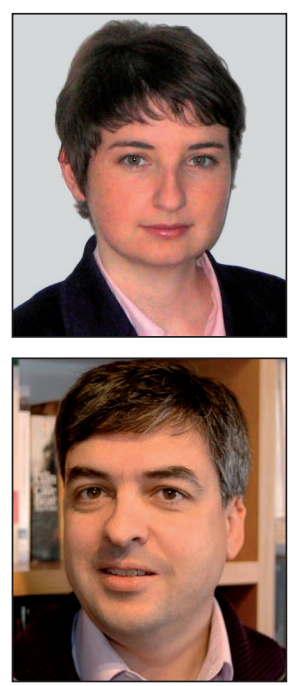

Naiara Sagarminaga-Gutiérrez es licenciada en documentación por la Universidad de Salamanca y magíster en sociedad de la información y el conocimiento por la Universitat Oberta de Catalunya. Entre 2007 y 2010 trabajó en Socialware SL, realizando análisis de opinión movilizada como infonauta. Actualmente trabaja como técnica de biblioteca en el Ayuntamiento de Portugalete.

Ayuntamiento de Portugalete, Biblioteca Municipal

Travesía de Salazar, s/n 48920 Portugalete, Bizkaia biblio12@portugalete.org

Agustí Canals-Parera es profesor de los Estudios de Ciencias de la Información y de la Comunicación de la Universitat Oberta de Catalunya. Licenciado y master en física por la Universitat Autònoma de Barcelona y doctor en administración y dirección de empresas (management sciences) por Esade (URL). Dirige el grupo de investigación KIMO sobre gestión de la información y el conocimiento en las organizaciones. Líneas de investigación: gestión estratégica del conocimiento, gestión de información, procesos de creación de conocimiento científico e innovación, redes sociales y sistemas complejos.

Estudis de Ciències de la Informació i de la Comunicació Universitat Oberta de Catalunya Rambla del Poblenou, 156. 08018 Barcelona acanalsp@uoc.edu

\section{Resumen}

La opinión movilizada y los medios sociales son una fuente de información muy valiosa para organizaciones e individuos en la toma de decisiones operativas y estratégicas como las relacionadas con la búsqueda de nuevos mercados, el seguimiento de las campañas de marketing o la mejora de la interrelación con el cliente. La aparición de organizaciones dedicadas a la monitorización de los medios sociales ha generado un campo de actuación para los profesionales de la información. La información procedente de esos medios debe ser recuperada y procesada para su utilización y es preciso crear sistemas automatizados de monitorización. Se analiza el proceso de recuperación y tratamiento de la opinión movilizada en internet como fuente para el estudio del clima social por parte de las organizaciones interesadas en ese tipo de conocimiento. El análisis se basa en las propiedades y dificultades específicas de ese tipo de opinión para concluir la necesidad de la participación de agentes humanos para la correcta evolución del proceso.

\section{Palabras clave}

Clima social, Opinión movilizada, Medios sociales, Recuperación de información, Tratamiento de información, Profesional de la información.

\section{Title: Mobilised opinion recovery and analysis for social climate research}

\begin{abstract}
Mobilised opinion and social media have become a valuable source of information for organisations and individuals in operational and strategic decision making processes, such as marketing campaigns, product monitoring and development, search for new markets or the improvement of customer relations. The emergence of social media monitoring companies has brought a new job market for information professionals, to the extent that this information has to be collected and processed, and new automated monitoring systems have to be developed. This article outlines a process of recovery and treatment of mobilised opinion on the internet as a source for social climate analysis by organisations. The specific characteristics and problems of this type of opinion are highlighted, which points to the need for the participation of human agents in the development of the process.
\end{abstract}

\section{Keywords}

Social climate, Mobilised opinion, Social media, Information retrieval, Information processing, Information professional. 
Sagarminaga-Gutiérrez, Naiara; Canals-Parera, Agustí. "Recuperación y tratamiento de opinión movilizada para el análisis del clima social". El profesional de la información, 2011, septiembre-octubre, v. 20, n. 5, pp. 510-515.

http://dx.doi.org/10.3145/epi.2011.sep.04

\section{Introducción}

La emergencia de los medios sociales (medios de comunicación basados en la interacción social) ha supuesto un cambio en la forma de comunicarnos y de acceder a la información (De-Gabriel-Eroles, 2010; Green, 2010). Entre los más populares podemos citar los blogs, microblogs (Twitter), wikis (Wikipedia), plataformas de redes sociales (Facebook) o de compartición de vídeos (YouTube).

Los trabajos realizados en torno a la socialización virtual (Castells, 2001; Hine, 2004) muestran que ésta tiene impacto no sólo en la sociedad virtual, sino también en la vida de las personas que forman parte de la misma. Los consumidores han encontrado en estos medios un lugar de gran riqueza en información y la posibilidad de compartir sus experiencias sobre marcas, productos y servicios (Zabin; Jefferies, 2008; Kozinets, 2002).

\section{En los medios sociales los consumidores encuentran información y pueden com- partir sus experiencias sobre marcas, productos y servicios}

La opinión movilizada es "el conjunto de percepciones y relaciones originadas por determinadas opiniones. Se trata de opiniones libres, expresadas sin ningún tipo de presión, creadas cuando y como al emisor le plazca" (Hernando-Guerrero, 2006). Al no responder a un cuestionario o entrevista, el medio garantiza al opinante la posibilidad de expresarse de acuerdo con sus prioridades, descubriendo aspectos desconocidos.

Frente a los sistemas automatizados basados exclusivamente en tecnologías como el data mining, el text mining y el data warehousing, la participación de un documentalista en el proceso incorpora el criterio de un profesional especialista. De esta forma se puede superar el simple análisis de textos, enriqueciéndolo con información sobre los vínculos y comportamientos de los opinantes.

Este artículo establece pautas para obtener la opinión movilizada relevante para el análisis del clima social de orga-

\section{Definiciones}

Minería de datos (data mining): extracción no trivial de información implícita en los datos.

Minería de textos (text mining): aplicación de las técnicas de data mining a los textos.

Organización de datos (data warehousing): provisión de herramientas y sistemas necesarios para la organización y extracción de información de los datos para la toma de decisiones (Han, 2006). nizaciones o individuos. Se hace particular hincapié en las particularidades que confieren a este tipo de opinión una elevada complejidad. Para los profesionales de la información este supone un nuevo campo de trabajo vinculado a las tecnologías de la información y la comunicación (TIC), así como a las nuevas formas de comercio, socialización y creación de información en la Red.

El artículo se estructura de la forma siguiente: tras introducir el concepto de análisis del clima social, se tratan sucesivamente las diferentes partes del proceso (recuperación, tratamiento y registro de la información) para finalizar con la presentación de las conclusiones.

\section{Análisis del clima social}

Se define clima social como "los aspectos humanos que pueden producir diferente graduación en una escala de calidez afectiva o social y de forma paralela a la climatología física [...]" (Uña-Juárez, 2004). El concepto de clima social se había aplicado fundamentalmente a centros escolares (por ejemplo, clima social escolar), hospitales y al desarrollo personal. Sin embargo las TIC (y específicamente internet) han propiciado un entorno virtual en el que el clima social adquiere relevancia y complejidad.

Moos (citado por Sánchez-Cánovas; Sánchez-López, 1994) afirma que los ambientes sociales tienen impactos distintos sobre las personas que viven y funcionan en ellos, estableciendo una causalidad recíproca entre individuos y ambiente. Por lo tanto, el interés de la opinión movilizada reside no sólo en la recogida y análisis de información, sino en la influencia de ésta en el entorno.

Las opiniones pueden ser analizadas en función de diversos campos, que deben ser seleccionados de antemano y corresponden a diferentes categorías de información, demarcando el clima social específico:

- campo geográfico: situación geográfica del opinante y/o del medio.

- campo idiomático.

- campo de opinante: conexión entre el objeto de análisis y el opinante.

- campo de servicio/producto.

- campo de medios: selección de los medios por su tipo.

La elección de campos se adecua a las necesidades del análisis, el mercado, el destino específico de la información y el servicio o producto. De esta forma, la elección del campo de opinante podría restringir el clima social a los usuarios de un producto o a aquellas personas que expresan su intención de utilizarlo pero que no lo hayan hecho aún (usuarios potenciales). El campo de medios podría restringir el uso de sitios web corporativos o de prensa, por considerar que las notas de prensa podrían sesgar los valores cualitativos. El uso de estas categorías no es excluyente, sino que deben combinarse y adaptarse para optimizar los resultados. 
Algunas de las posibles aplicaciones prácticas de este tipo de análisis son: la comparación del clima social de diferentes personas para elegir a un posible cargo honorífico, excluyendo los opinantes de la propia organización; el seguimiento de un producto, restringido a los usuarios y usuarios potenciales; o el análisis del clima social de una organización, previo a una fusión, centrándose en los medios nacionales.

La información a recuperar y procesar puede encontrarse en varios formatos y debe combinarse la investigación cuantitativa y la cualitativa para la obtención de conclusiones válidas. Estas conclusiones, al ser extraídas en un proceso específico para un caso determinado, no pueden ser extrapoladas más allá del objeto de análisis concreto.

\section{Recuperación de la información}

El primer paso en la recuperación de información es la definición de la estrategia de búsqueda. Ésta resultará más eficaz cuanto mejor se defina lo que se busca. El proceso habrá de ser planificado cuidadosamente antes de comenzar. Durante su ejecución deberán establecerse mecanismos de comprobación de la calidad de los resultados que se van obteniendo y de valoración de su relevancia (Fuentes, 2001). Todo ello es necesario para asegurar la eficiencia de las búsquedas de opinión movilizada y su principal propósito: la representatividad de los resultados. Fuentes (2001) propone un esquema de toma de decisiones basado en preguntas que el profesional debe hacerse en cada una de las fases (tabla 1).

Una vez establecida la estrategia puede empezar el proceso de búsqueda propiamente dicho. El primer condicionante, y uno de los rasgos característicos de la opinión movilizada, es el alto grado de libertad del opinante a la hora de expresarse: vocabulario ilimitado, sinonimia y polisemia, uso de giros y expresiones propias y coloquiales, aparición de errores ortográficos, uso de figuras lingüísticas, etc.

El primer condicionante es el alto grado de libertad del opinante para expresarse
Por otro lado, la búsqueda está condicionada por el tipo de medio donde se realiza. La opinión movilizada no se expresa de la misma manera en un foro, que en una plataforma de red social como Facebook, en Twitter o en un portal de opinión. Algunos medios disponen de sistemas de búsqueda integrados específicamente creados para los mismos, que facilitan la recuperación, haciendo posible recuperar mensajes individuales o temas completos, con un alto nivel de exhaustividad.

Fuentes de utilidad son los directorios (Open Directory Project), los buscadores (Google) y los metabuscadores generalistas (Metacrawler). Los primeros pueden ser convenientes para realizar una primera lista de medios para cada clima social en función de su tema, mientras que los segundos favorecen encontrar información específica, actualizada e indizada a niveles detallados.

Una ecuación de búsqueda compleja es la formada por dos términos y un operador (Baeza-Yates; Ribeiro-Neto, 2010; Ferran; Pérez-Montoro, 2009). Los principales operadores son: booleanos, truncamientos, proximidad y rango. En los buscadores y metabuscadores, mediante la ecuación de búsqueda compleja, los inconvenientes de sinonimia y polisemia pueden ser minimizados restringiendo o asociando determinados términos mediante operadores booleanos.

\section{La búsqueda está condicionada por el tipo de medio donde se realiza}

Como es sabido, los operadores de proximidad son útiles en caso de nombres compuestos, y para discriminar un determinado producto o servicio que esté comercializado por más de una organización. Los truncamientos sirven para recuperar opinión de diferentes productos con una misma raíz en su nombre, agilizando el proceso.

Algunas utilidades que pueden mejorar los resultados como la acotación de fecha, dominio o idioma. El uso de las comillas es útil para la búsqueda de personas, tanto en orden directo como inverso del nombre y apellidos.

Entre las fuentes interesantes para la determinación del clima social destacan las comunidades o portales de opinión (Ciao), destinadas a que los internautas compartan sus experiencias en torno a productos, marcas y servicios. Poseen sencillos sistemas de búsqueda y tienen un gran volumen de información. Los opinantes encuentran aquí un soporte donde establecer complejos vínculos de confianza y colaboración, lo que constituye un entorno muy útil para el análisis del clima social. La opinión movilizada sólo corresponde a aquella parte de la información que se expone libremente, eliminando los cuestionarios y baremos comunes en estos medios.

Las redes sociales son, cada vez más, otra fuente de opinión movilizada, pues

Tabla 1: Decisiones relevantes en la búsqueda de información (Fuentes, 2001) 
contienen gran cantidad de páginas personales y sistemas de mensajería pública donde los miembros exponen su opinión. Las políticas de privacidad son una de las limitaciones fundamentales para la obtención de información, puesto que los opinantes pueden restringir su visualización a aquellas personas que se encuentran en su red de contactos. También se habrá de considerar la presencia de formatos no textuales, como montajes fotográficos y vídeos, que presentan mayor dificultad para su recuperación.

Los medios de uso restringido y la opinión movilizada que no está presente en ningún sistema de recuperación son: foros de acceso mediante password, perfiles de Facebook cuya privacidad no permite el acceso fuera de la lista de amigos, pequeños blogs que no han sido encontrados por los buscadores, etc. Es necesario recuperar un volumen de documentos (una muestra) que sea representativo del conjunto de la opinión, teniendo en cuenta la imposibilidad de conocer el volumen exacto de información existente.

Una vez obtenida la información se inicia su análisis. Para este proceso se contrastan los documentos con los campos seleccionados para el clima social, comprobando la adecuación del idioma e identificando el vínculo entre el opinante y el objeto de análisis. Posteriormente se realiza el análisis del medio y la identificación de la extensión del dominio, que servirá para determinar alguna orientación si la tuviera. Los datos de autoría se pueden obtener de las secciones "Sobre el autor", "Acerca de esta página" o perfiles públicos. Además esta identificación favorece la selección de documentos atendiendo a los campos de medios y geográfico.

\section{Tratamiento de la información}

La indización es, según la UNE 50-121-1991 (Aenor, 1991), "la acción de describir o identificar un documento en relación con su contenido". Es aplicable al sistema de tratamiento de opinión movilizada, por compartir el proceso de asignación de términos para la representación de su contenido. Según esta misma normativa, el proceso de indización consta de tres fases:

- examen del documento y definición de su contenido;

- identificación y selección de las nociones del contenido;

- selección de los términos de indización que van a representar a esos conceptos.

La indización de la opinión movilizada está orientada a la obtención de todos los aspectos presentes en la opinión. En ocasiones los documentos analizados no presentan una gran extensión, pese a lo cual pueden proveer gran cantidad de información (vínculos con otros autores u opiniones, expresiones de expectación o confianza...).

Esta información se indexa mediante un lenguaje controlado. La utilización de lenguaje libre podría tener como consecuencia una pérdida para el análisis cuantitativo de la opinión movilizada y un gran volumen de términos podría resultar ineficaz para el análisis del clima social.

Hay que tener en cuenta que cada clima social es específico. Por lo tanto, aunque costoso, es aconsejable la construcción de un tesauro que establezca tanto los términos de indización como las conexiones entre los mismos, presentando un entramado complejo y completo de los conceptos presentes. Los tesauros no creados específicamente para un clima social resultan ineficaces debido a que no pueden reflejar correctamente la conceptualización de los opinantes.

La UNE 50-106-90 (Aenor, 1990) establece las directrices para la construcción de tesauros monolingües. La extracción de términos a partir de un número limitado de documentos servirá para elegir los descriptores y no descriptores y establecer las conexiones entre los mismos.

El análisis del clima social intenta observar no sólo la opinión movilizada, sino también los vínculos establecidos en torno a ella. Pese a que en los tesauros no suele ser común la relación asociativa, ésta es fundamental para nuestros objetivos, facilitando la comprensión de las conexiones que hacen los opinantes entre los diferentes conceptos. Cuando los términos no tienen una relación jerárquica se asocian de forma sintagmática (Lancaster, 2002).

\section{Se intenta observar no sólo la opinión} movilizada, sino también los vínculos establecidos en torno a ella

Una vez obtenido el lenguaje controlado se comienza la asignación de los descriptores, considerando únicamente aquella información que el opinante haya expresado libremente. Han de eliminarse las citas, que deben ser analizadas como opiniones independientes, excepto cuando la opinión tenga como finalidad sustentar las mismas.

Consignar los datos de los campos asociados a cada uno de los términos utilizados aporta un mayor nivel de definición, un valor añadido. Cada uno de éstos será individualizado para cada descriptor utilizado.

La opinión movilizada se caracteriza por poseer cualidad, considerada siempre desde el punto de vista del opinante. Esta cualidad habrá de ser representada junto a descriptores y campos para medir la negatividad presente en el clima social. Un tercer aspecto se refiere a la cualidad neutra de la opinión, constituida por las comunicaciones meramente informativas.

A partir del tesauro y de los documentos recuperados da comienzo el proceso de análisis de los textos, que corresponde a una indización completa de las opiniones presentes, la obtención de la información no trivial y el almacenamiento de dicha información con objeto de hacerla accesible. Aquí confluyen el data mining y text mining (minería de datos y minería de textos) como métodos de análisis no automatizados.

El procedimiento expuesto no tiene un orden secuencial, sino que puede ser realizado de forma simultánea al ser aplicado a los documentos recuperados.

Para el análisis de la opinión movilizada se procede al minado de los textos, de forma que se obtenga una representación de los mismos basada en un lenguaje controlado. Éste sirve para evitar las complicaciones y ambigüedades producidas por el lenguaje natural y facilita el posterior análisis. La 
problemática de la opinión movilizada y las limitaciones de la indización por extracción hacen que el minado de textos no automatizado ofrezca un valor añadido a los datos obtenidos. Toda esta información habrá de ser convenientemente ordenada en bases de datos o tablas para su recuperación y análisis.

Posteriormente se realiza un proceso de minería de datos también de forma no automatizada. La información obtenida es de utilidad para el análisis del clima social, trascendiendo las limitaciones del proceso automatizado, al ser la persona que ha realizado la minería de textos (y que tras dicho proceso posee una gran cantidad de información de valor añadido), la que extrae la información no trivial.

Hay que resaltar la necesidad de emparejar los campos y la cualidad anteriormente expuestos con los datos obtenidos de la minería de datos y de la minería de textos. La cuantificación de dichos campos será una parte fundamental del análisis del clima social, analizando la afinidad entre temáticas y campos presentes en la opinión movilizada.

Por último, el data warehousing es el proceso semi-automatizado por el que los datos obtenidos en los dos pasos anteriores son organizados y almacenados en un soporte digital.

\section{Registro de la información}

El registro es la última información a consignar en el proceso de análisis de la opinión movilizada. Los datos identificativos indispensables son el localizador y la fecha del documento.

El localizador deberá ser lo más específico posible, siendo recomendable optar por el que enlace de la forma más directa con el documento. La fecha de éste debe consignarse con el fin de facilitar la recuperación y la temporización del clima social, para que pueda ser analizado en su conjunto a lo largo de un período de tiempo y conocer su evolución.

Adicionalmente se puede guardar una copia del documento mismo. Internet es un medio dinámico donde los medios sociales surgen y desaparecen con relativa rapidez, por lo que conservar una copia nos asegura tenerlo siempre disponible.

El medio en que está presente la opinión movilizada es un aspecto de relevancia para el análisis del clima social. Añadir datos sobre la visibilidad, tipo o dinamismo del medio, entre otros, facilitan la comprensión de la importancia y participación de los opinantes. Sitios como Alexa Reach, que ofrece el número de usuarios que visitan un sitio, facilitan este tipo de información.

\section{Conclusiones}

El valor de la información en los medios sociales es indiscutible. La emergencia de empresas destinadas a su análisis (Social Target, 2008; Zabin; Jefferies, 2008) y los trabajos de investigación aparecidos en los últimos años lo corroboran. Estas investigaciones demuestran claramente el valor de dicha información, haciendo hincapié en sus posibles aplicaciones en el marketing, la toma de decisiones y la relación con los clientes. Cabe destacar los estudios sobre netnogra- fía, o etnografía de comunidades virtuales (Turpo-Gebera, 2008). Sin embargo, las obras sobre la recuperación y el procesamiento de opinión movilizada para el análisis del clima social son aún inexistentes.

A lo largo de este artículo se han presentado unas pautas generales que servirán para la recuperación de opinión movilizada de diversos medios sociales. Así, se ha visto cómo las estrategias de búsqueda complejas mejorarán los resultados. Además, hay que considerar la diversidad de medios en los que está presente la opinión movilizada, lo que requiere estrategias de búsqueda específicas para cada uno. Finalmente, además de los buscadores y directorios generales habrán de utilizarse los específicos de cada medio, con diferentes posibilidades de búsqueda.

Es conveniente disponer de una lista de buscadores y directorios con un gran número de medios donde elegir los más adecuados para cada clima social. La diversidad de materias presentes en los medios sociales tiene como consecuencia que la lista de medios sea dinámica, por lo que habrá de ser actualizada constantemente.

Los documentos obtenidos mediante estrategias de búsqueda serán seleccionados de acuerdo con los campos acordados en función de los intereses de la organización o individuo objeto del análisis del clima social.

Evidentemente, los sistemas de búsqueda automatizados son muy útiles, pero puesto que el volumen de opinión recuperado debe ser representativo del conjunto, la revisión manual de medios puede resultar también una fuente de información fundamental.

Una vez recuperada la información ha de procederse a su tratamiento y registro. Entre las diversas alternativas, usar un lenguaje controlado para la indización del contenido permite representarlo de forma sintética y accesible. Mediante la creación de un tesauro especializado es posible luego obtener información detallada, integrada tanto por la opinión movilizada como por el contexto y el medio que son objeto del análisis del clima social.

Por otro lado, las limitaciones de los sistemas automatizados, las dificultades derivadas del uso del lenguaje natural y la jerga usada en internet (emoticonos, escritura tipo sms, etc.), muestran que la participación de un profesional de la información resulta imprescindible para la obtención de datos representativos y exhaustivos.

\section{Bibliografía}

Aenor (Asociación Española de Normalización y Certificación). UNE 50-106-90: Directrices para el establecimiento y desarrollo de tesauros monolingües. Madrid: Aenor, 1990.

Aenor (Asociación Española de Normalización y Certificación). UNE 50-121-91: Documentación. Métodos para el análisis de documentos, determinación de su contenido y selección de los términos de indización. Madrid: Aenor, 1991.

Baeza-Yates, Ricardo; Ribeiro-Neto, Berthier. Modern information retrieval: the concepts and technology behind search. 2a ed. New York: Addison Wesley, 2010. ISBN: 9780321416919 
Castells, Manuel. La era de la información: economía, sociedad y cultura. Madrid: Alianza, 2001. ISBN: 8420642460

De-Gabriel-Eroles, Josep-Lluís. Internet marketing 2.0: captar y retener clientes en la Red. Barcelona [etc.]: Reverté, cop. 2010, ISBN 9788429126341

Ferran, Núria; Pérez-Montoro, Mario. Búsqueda y recuperación de la información. Barcelona: Editorial UOC, 2009, ISBN: 9788497888349

Fuentes, Marta. "Naufragar en internet. Estrategias de búsqueda de información en redes telemáticas". Virtual Educa, 2001,

http://www.uoc.edu/web/esp/art/uoc/0109037/fuentes. html

Green, Leila. The internet: an introduction to new media. New York: Berg, 2010, ISBN: 9781847882998

Han, Jiawei; Kamber, Micheline. Data mining: concepts and techniques. San Francisco: Morgan Kaufmann, 2006. ISBN: 1558604898

Hernando-Guerrero, Alfonso. Nuevo concepto: opinión movilizada.

http://www.komunika.info/?p=162

Hine, Christine. Etnografía virtual. Barcelona: Editorial UOC, 2004. ISBN: 8497880196

Kozinets, Robert V. "The field behind the screen: using net- nography for marketing research in online communications". Journal of marketing research, 2002, February, v. XXXIX, pp. 61-72.

http://www.nyu.edu/classes/bkg/methods/netnography. $p d f$

Lancaster, Frederick-Wilfrid et al. El control del vocabulario en la recuperación de información. Valencia: Universitat de València, 2002, ISBN: 8437054443

Sánchez-Cánovas, José; Sánchez-López, María-del-Carmen. Psicología diferencial: diversidad e individualidad humanas. Madrid: Ramón Areces, 1994, ISBN: 8480041390

Social Target. Guide to social media analysis http://www.socialtarget.com/research/guide.html

Turpo-Gebera, Osbaldo-Washington. "La netnografía: un método de investigación en internet". Revista iberoamericana de educación, 2008, octubre, n. 47/2.

http://www.rieoei.org/deloslectores/2486Gebera.pdf

Uña-Juárez, Octavio; Hernández-Sánchez, Alfredo. Diccionario de sociología. Madrid: ESIC Editorial, 2004. ISBN: 9788473563598

Zabin, Jeff; Jefferies, Alex. Social media monitoring and analysis: generating consumer insights from online conversation. Boston: Abeerden Group, 2008.

http://www.aberdeen.com/summary/report/benchmark/ 4587-RA-consumer-insights-online.asp? $r s s=y$

\section{ProQuest}

\section{¡NUEVO! ProQuest Business Package}

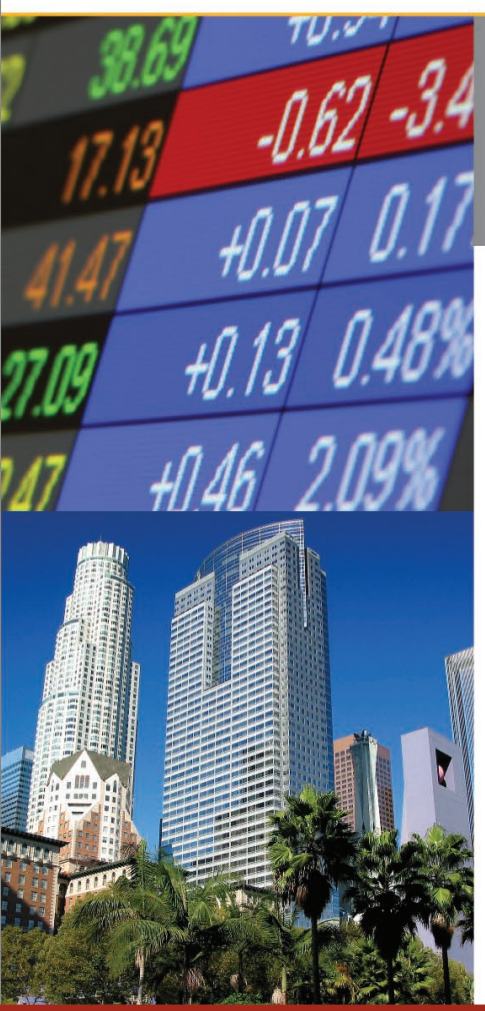

Más recursos para su biblioteca con un ahorro de hasta el $20 \%$.

Combinando las 6 mejores bases de datos de ProQuest en el área de negocios y gestión, la nueva colección ProQuest Business Package le ofrece más de 5.000 publicaciones periódicas junto con una colección única de contenido no periódico y otros tipos de documentos totalmente nuevos. 